Research Article

\title{
Investigation of Flow Control for the Hypersonic Inlets via Counter Flow
}

\author{
Xiaoqiang Fan and Yuan Tao \\ Science and Technology on Scramjet Laboratory, National University of Defense Technology, Changsha 410073, China
}

Correspondence should be addressed to Xiaoqiang Fan; xiaoqiangfan@hotmail.com

Received 1 April 2015; Accepted 4 June 2015

Academic Editor: Linda L. Vahala

Copyright (C) 2015 X. Fan and Y. Tao. This is an open access article distributed under the Creative Commons Attribution License, which permits unrestricted use, distribution, and reproduction in any medium, provided the original work is properly cited.

Experimental results show that there exist two flow fields in the hypersonic inlets when the forebody waves interact with the lip boundary, which is similar to the shock reflection ion hysteresis phenomenon. In order to improve the performance of the flow field, counterflow is applied to control the shock reflection configuration in the hypersonic inlets. For better understanding of the internal mechanism, inviscid numerical simulation is conducted. And the results demonstrate that it is feasible to realize the transition between the regular reflection configuration and the Mach reflection ion configuration in the hypersonic inlets. That is because the von Neumann criterion and detached criterion play a dominant role, respectively, in these transitions. In addition, the evolution process of Mach reflection ion in the hypersonic inlets can be divided into three stages: transmission of waves, emergence of Mach stem, and stabilization of flow field.

\section{Introduction}

As the X-43 operated successfully in March 2004, the issues encountered in the scramjet engines have been an area of intense investigation, like the hypersonic inlets. Further, in the investigation of the hypersonic inlets, it is extremely critical to improve the inlets' performance for capturing and compressing the airflow more effectively, as discussed by Segal [1] .

In engineering practice, if the flight Mach number of the inlets is beyond the design Mach number or the angle of attack flight exceeds its rated value, the waves generated by forebody may spread into the lip. These waves interact with the lip boundary layer to show two different flow fields, shown in Figure 1. One is a started flow field and the other is an unstarted flow field, displayed in frames (a) and (b), respectively. In the started flow field, the forebody waves reflect over the lip without separation, while, in the unstart flow field, a new wave configuration similar to Mach reflection configuration wave in structure forms after the interaction between the forebody waves and the lip boundary layer. It is noteworthy that the new wave configuration is different from the Mach reflection, because of the existence of the separation.

Further analysis indicates that the reasons for the dual solution flow field may be related to the shock reflection hysteresis phenomenon. Ben-Dor [2] points out that there exist two kinds of shock reflection configurations in steady hypersonic flows. One is named regular reflection (RR) and the other is called Mach reflection (MR). The fine structures of above shock reflections are displayed in Figure 2 by Tao et al. [3]. Generally speaking, the regular reflection shown in Figure 2(a) benefits to establish a durable and stable flow field which can ensure the proper function of the hypersonic inlets, while the Mach reflection configuration shown in Figure 2(b) may have a negative effect on the hypersonic inlets' performance; that is, the total pressure loss remains high, and the flow captured by the inlets decreases acutely. In other words, appropriate shock reflection configurations are beneficial for hypersonic inlets to capture and compress airflow clearly. Moreover, Hornung et al. [4] indicate that both $R R$ and $M R$ are physical under the same flow condition in the dual solution domain, which is similar to the phenomenon mentioned above. Thus, for better understanding of controlling the complex wave configurations in the hypersonic inlets, it is essential to take appropriate measures to control the shock reflection configurations, especially when they have two or more solutions.

Aiming at this problem, extensive work has been carried out. According to the previous research results, the flow 


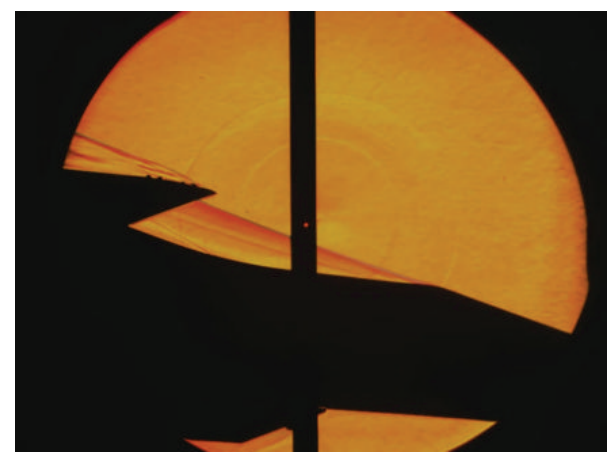

(a) Started flow field

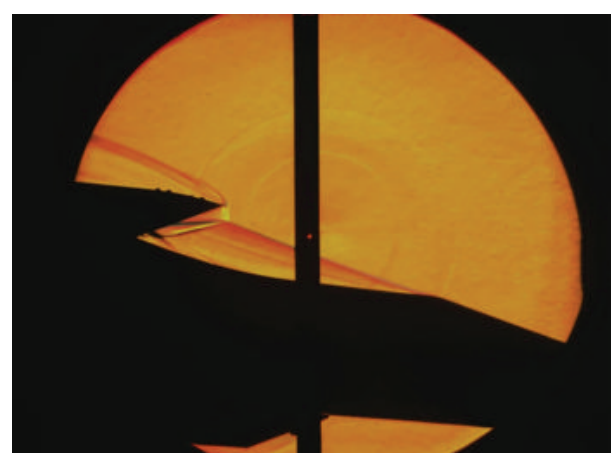

(b) Unstarted flow field

FIGURE 1: Schlieren visualisation of an interaction between the forebody waves and the lip boundary layer in the hypersonic inlets.

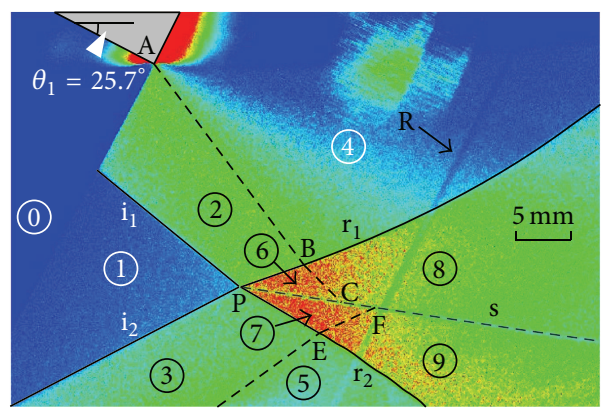

(a)

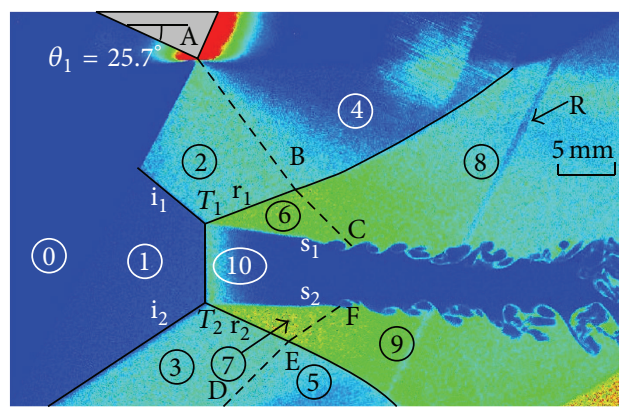

(b)

FIGURE 2: The nanotracer planar laser scattering images of the shock reflection configurations illustrated by Tao et al. [3]: (a) regular reflection $(\mathrm{RR})$ and (b) Mach reflection (MR).

control methods can be divided into three types mainly. Firstly, variable geometry design is well applied for controlling the shock reflection configurations in the hypersonic inlets. Chpoun et al. [5] and Ivanov et al. [6,7] indicate that changing the shock generator's angle is an effective way to achieve the transition between RR and MR. Based on this point, Tam et al. [8], Moss and Perrell [9], and Falempin et al. [10] put forward several methods to control the wave configurations for improving the inlets' performance. However, the mechanical adjusting devices which are comparatively complex may increase inlets' structural weight evidently.

Secondly, the energy addition control technique is implemented to configure appropriate shock reflection configurations. Specifically, laser induced optical breakdown has been demonstrated as a method for local modification of the shock reflection structure in supersonic flows by Yan et al. [11-13] and Adelpen et al. [14]. Besides, active control technique based on virtual shapes created by energy addition upstream of the inlet throat is proposed by Shneider et al. [15]. Moreover, Lapushkina et al. [16] investigate the relationship between electric and magnetic fields and the shock wave configurations in hypersonic inlets. However, energy system maintains quite high requirement in this way.

Thirdly, boundary layer suction is well applied for flow control in hypersonic inlets. It is important to note that the flow field has high flow loss, during the suction, despite the fact that it is an effective way to realize the transition between two different shock structures [17].

Also, the physical mechanism about the wave configurations shown in Figure 1(b) is still unknown. Hence, considering the complexity of the viscous flow field, it is extraordinarily meaningful to investigate the inviscid situation, which is the foundation of the viscous analysis. Based on the inviscid precondition, there is no separation in the inlets, and the main problem needing more attention is the shock reflection configurations. Also, this paper investigates new methods to control the shock reflection configurations in the hypersonic inlets via counterflow, especially when the shocks are in the dual solution domain, and explains the inherent mechanism in substance, combining correlative theory.

\section{Numerical Approach}

The results of this paper are achieved by solving the Euler equations numerically. The Euler computations are performed using the finite-volume technique to solve the governing equations of flow, based on the assumption that the shock reflection configurations in the hypersonic inlets, which are induced by the interaction of shock waves, are independent of the viscous effects. Also, the equations are discretized in space and time following the control volume approach and utilizing a density-based explicit solver. 


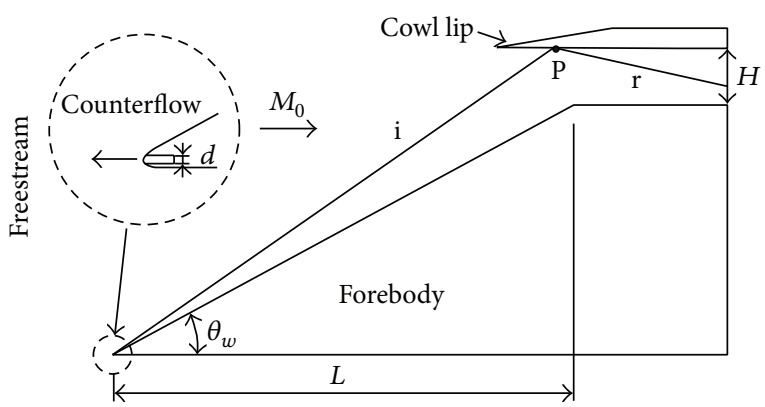

Figure 3: A simplified physical model for computation.

A second-order upwind scheme is used to discretize the convective terms. Ideal gas model of air with the specific heat ratio $\gamma=1.4$ was employed in this paper. Besides, time step size of the calculation was $1 \times 10^{-8} \mathrm{~s}$ for the unsteady process.

To further investigate the inherent mechanism of local modification of the shock reflection structures in the hypersonic inlets via counterflow, a simplified physical model is abstracted in this paper, as shown in Figure 3. Specifically, a single-ramp inlet at $\theta_{w}$ is considered to be installed into a uniform hypersonic flow at $M_{0}$. The inflow pressure and temperature which are kept constant are set at $p_{0}, T_{0}$, respectively, and the extrapolation technique was used for outlet. It is noteworthy that there exists a slot which is used to add the counterflow at the front of the wedge, and the width is set as $d$.

Ben-Dor [2] indicates that the shock reflection configuration is determined by the flow Mach number $M_{0}$ and the ramp angle $\theta_{w}$, together. According to this conclusion, domains of possible shock wave reflection wave configurations can be presented in the $\left(M_{0}, \theta_{w}\right)$-plane, shown in Figure 4. In the picture, dashed line and solid line denote, respectively, the detached condition and the von Neumann condition. They divide the entire $\left(M_{0}, \theta_{w}\right)$-plane into three domains: Mach reflection domain $\mathrm{A}$, dual solution domain $\mathrm{B}$, and regular reflection domain C. Dual solution domain is a domain inside which both $\mathrm{RR}$ and $\mathrm{MR}$ wave configurations are theoretically possible. Thus, domain B is clearly critical for controlling the shock reflection configurations in the hypersonic inlets. Based on above reasons, $M_{0}$ and $\theta_{w}$ are set at 6 and $22^{\circ}$, which is inside the dual solution domain. Also, $H / L, d / L$ are $0.2,0.003$. Besides, on the assumption that the hypersonic inlets work at an altitude of $26 \mathrm{~km}, p_{0}$ and $T_{0}$ can be set at $2188.08 \mathrm{pa}$ and $222.58 \mathrm{~K}$, respectively.

To ensure the accuracy of the results, it is essential to construct the computational grid with high orthogonality and smoothness. Also, the mesh is refined based on a pressure gradient criterion in steady cases, and the total number of cells in steady computation is 200000, while that in unsteady computation is 40000 . Figure 5 gives the Mach number distribution along the cowl lip. Relatively speaking, the numerical results (200000 cells) are in close agreement with the theoretical value.

\section{Results and Discussion}

A new flow control method for realizing the transition between RR and MR mutually is put forward. In this method,

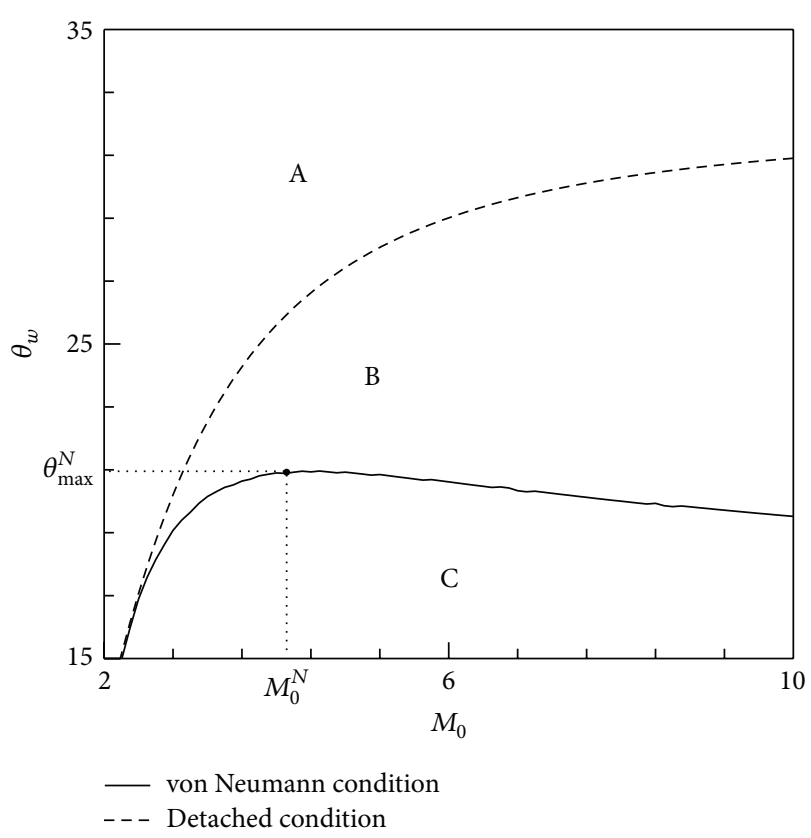

FIGURE 4: Domains of possible shock wave reflection wave configurations in the $\left(M_{0}, \theta_{w}\right)$-plane.

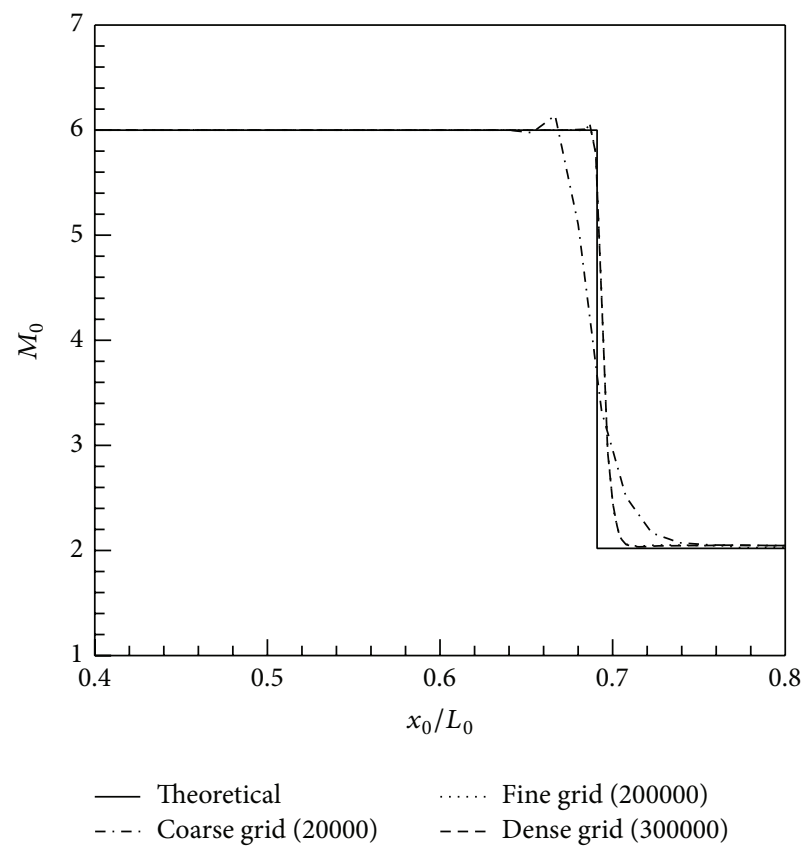

FIGURE 5: The comparison between numerical and theoretical results.

the counterflow is well applied in altering the upstream shock shape and then promotes the formation of the appointed shock reflection configuration. The results are discussed as below.

3.1. Counterflow. In order to further understand the internal mechanism, this paper pays more attention to the wave configurations affected by counterflow, firstly. Figures 6 and 7 


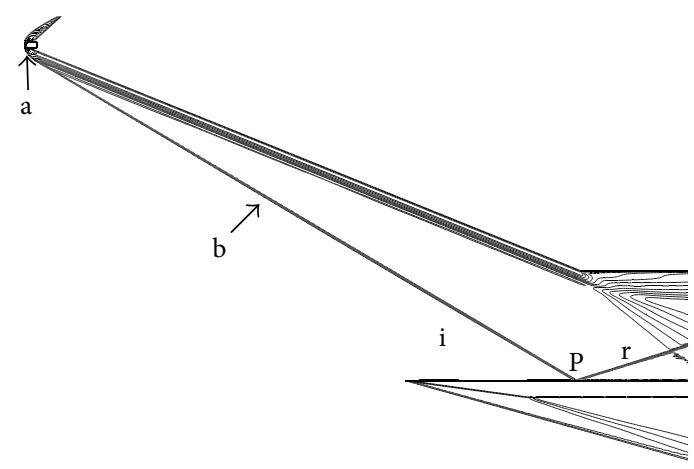

Figure 6: The flow field without counterflow.

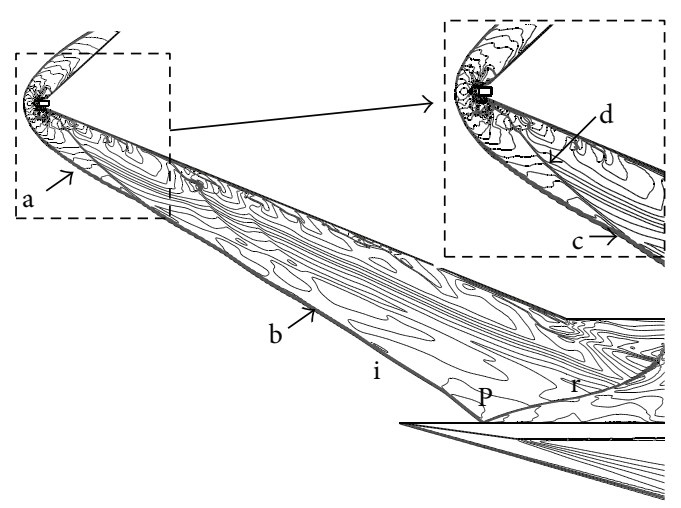

FIgURE 7: The flow field affected by counterflow.

give the flow fields without or with the effects caused by counterflow, respectively. Further analysis indicates that these wave configurations both consist of an incident shock i and a reflected shock $r$. Besides, a detached shock which is denoted by $\mathrm{a}$ and an attached shock which is pointed by $\mathrm{b}$ constitute the incident shock, together.

However, there are some differences in the details. Specifically, the detached shock a shown in Figure 7 which is induced by counterflow is larger in scale than that shown in Figure 6. Besides, the attached shock b shown in Figure 7 which is affected by counterflow keeps light curving, while that in Figure 6 remains straight. Also, the angle of the curving shock is larger than the straight one. This is because there exists a compression wave $d$ which is induced by counterflow to increase the shock wave angle, and the interaction point is the junction $\mathrm{c}$. It also leads to the phenomenon that the reflection point $\mathrm{P}$ moves upstream and the shape of reflected shock $r$ changes correspondingly.

Figure 8 illuminates the flow field near the slot after local amplification. In the picture, wave a and wave d denote the detached shock and the compression shock, respectively, which are mentioned above. Wave e denotes the fan-shaped shock which is induced by the counterflow. Besides, wave a and wave e divide the region into three parts, that is, freestream depression region $\mathrm{A}$, high-pressure region $\mathrm{B}$, and jet depression region $\mathrm{C}$.

Further analysis indicates the velocity and the total pressure of the counterflow are key parameters for better

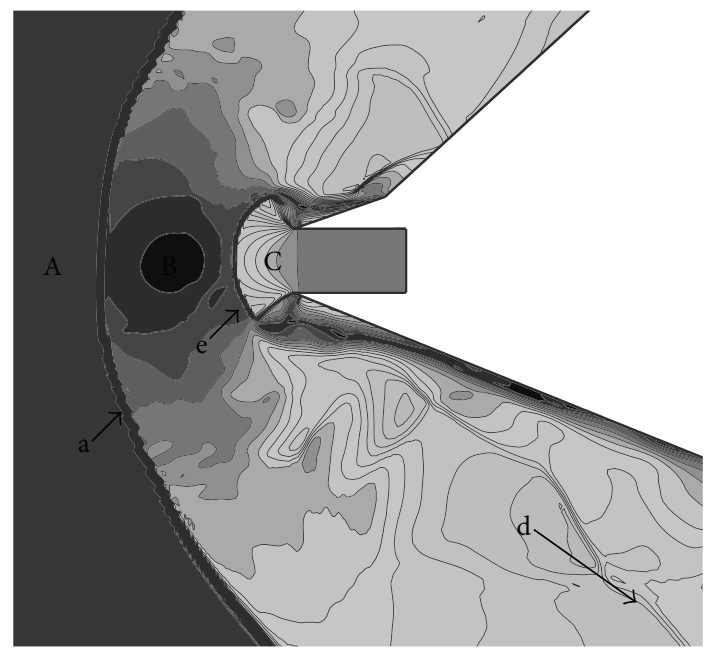

FIGURE 8: The flow field after partial enlargement.

understanding of the relationship between the counterflow and the flow field. On the one hand, increasing the total pressure of the counterflow results in the phenomenon that the detached shock a grows larger in scale and the junction c moves downstream, when the velocity of the counterflow remains fixed. On the other hand, increasing the velocity of the counterflow has little effect on the wave configuration of the detached shock a. However, the reflection point $\mathrm{P}$ moves downstream as the velocity grows, when the total pressure is fixed, shown in Figure 9.

3.2. Flow Control Process. The numerical results disclose that adding counterflow at the leading edge of forebody is an effective way to realize the transition between RR and MR mutually. Thus, this paper gives the whole flow control process for better understanding of the internal mechanism, shown in Figure 10.

From Figure 10(a), the initial wave configuration is RR configuration. At this moment, counterflow is added to change the shock structures, which is represented by symbol A. In addition, the total pressure and the velocity of the counterflow are $140000 \mathrm{pa}$ and $300 \mathrm{~m} / \mathrm{s}$, respectively. Soon after that, a detached shock emerges near the slot and interacts with the oblique shock, such that the shock angle at the junction c decreases, shown in Figure 10(b). Gradually, the disturbance of counterflow spreads downstream, which is represented by symbol B. In Figure 10(c), RR configuration still exists in the flow field. However, once the counterflow is turned off, the MR configuration emerges after stabilization, shown in Figure 10(d). Thus, the unilateralism transition from $R R$ to $M R$ is realized via counterflow.

In the same way, counterflow is added to change the shock configuration, which is represented by symbol D. However, the total pressure and the velocity of the counterflow are reset as $110000 \mathrm{pa}$ and $100 \mathrm{~m} / \mathrm{s}$, respectively. Persistent disturbances (symbol E) have a great impact on the shock structure, in company with the spread of waves. Juxtaposing Figures $10(\mathrm{~d})-10(\mathrm{f})$, it is evident that once the reflection point $\mathrm{P}$ is affected by counterflow, the height of Mach stem descends 

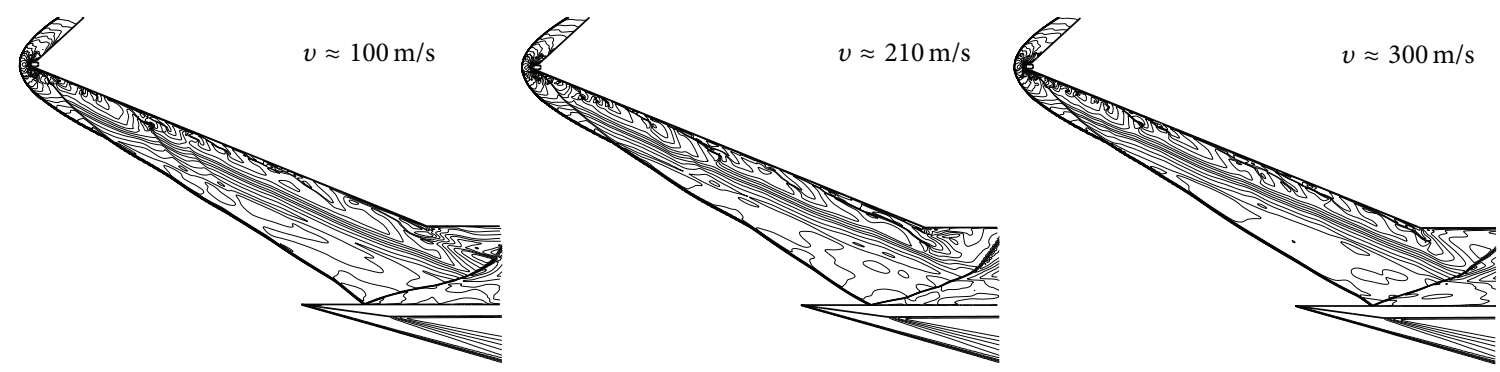

FIgURE 9: Three flow fields in the different velocities of counterflow when the total pressure is fixed.

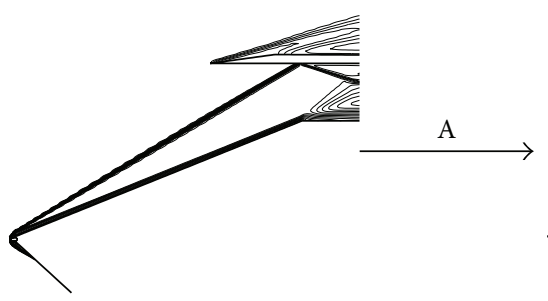

(a) $t=0 \mathrm{~s}$

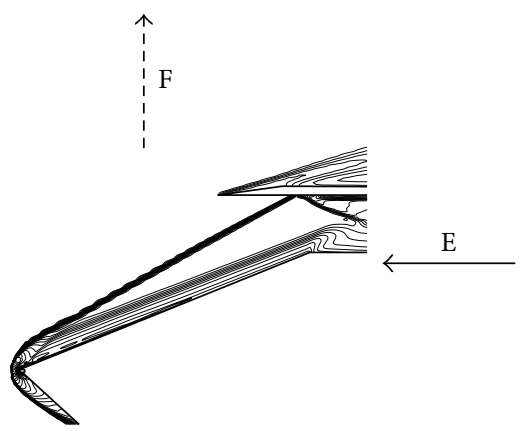

(f) $t=2.66 \times 10^{-3} \mathrm{~s}$

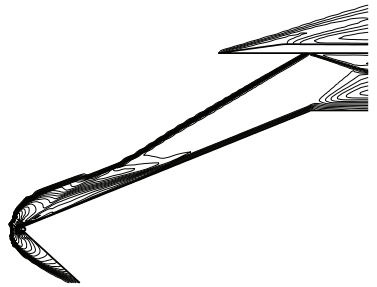

(b) $t=2.00 \times 10^{-4} \mathrm{~s}$

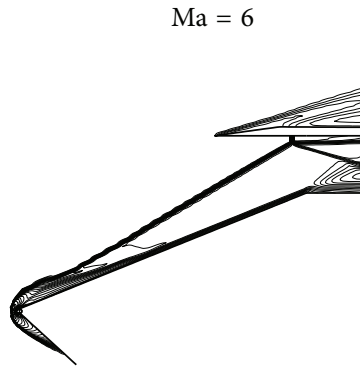

(e) $t=1.94 \times 10^{-3} \mathrm{~s}$
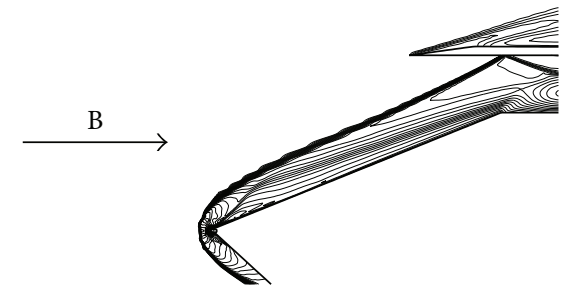

(c) $t=4.77 \times 10^{-4} \mathrm{~s}$

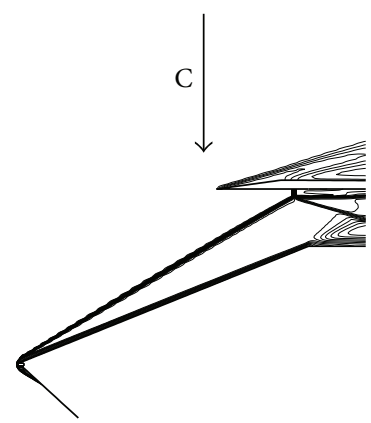

(d) $t=1.76 \times 10^{-3} \mathrm{~s}$

FIgURE 10: The flow control process.

rapidly, until it disappears. Also, a RR configuration shown in Figure 10(f) is obtained. Then, the counterflow is turned off which is denoted by symbol F. After stabilization, the shock reflection configurations shown in Figure 10(a) can be obtained again, finally.

The inviscid numerical results mentioned above display the unsteady flow control process in detail. In conclusion, by adding the counterflow, it is possible to realize the bidirectional transition between $\mathrm{RR}$ and $\mathrm{MR}$ configuration in the hypersonic inlets.

\subsection{Discussion for Reasons. By analyzing the structural} changes of the flow field induced by counterflow further, this paper also explains the internal reasons for the bidirectional transition, combining with shock reflection hysteresis theory. Aiming at this problem, 55 moments in the flow control process are selected. Then, the shock angle of the incident shock $i$ at reflection point $P$ and the height of Mach stem are measured at each moment. The results were displayed in Figure 11.

Figure 11 illustrates the shock angle of incident shock $i$ at the reflection point $\mathrm{P}$ along the time and describes the height alteration of Mach stem during the flow control process. In Figure 9, horizontal line a and horizontal line c denote the detached shock $\alpha^{D}\left(M_{0}\right)=39.8^{\circ}$ and the von Neumann angle $\alpha^{N}\left(M_{0}\right)=28.9^{\circ}$ at $M_{0}=6$, respectively, which indicates there exist two different solutions of shock reflection pattern in the hypersonic inlets when the flow condition is inside the region between the two lines. Also, the forebody shock angle $\alpha_{w}\left(M_{0}\right)$ can be obtained by applying the oblique shock wave equations, which is symbolized by horizontal line $b$. And the value is $30.6^{\circ}$. In addition, the capital letters which stand for the vertical dashed line are corresponding to that in Figure 10.

From Figure 11, the initial incident shock angle at reflection point $\mathrm{P} \alpha_{\text {ref }}\left(M_{0}\right)$ is the forebody shock angle $\alpha_{w}\left(M_{0}\right)$. Also, $\alpha_{\text {ref }}\left(M_{0}\right)$ remains invariant in a short period $\left(\mathrm{k}_{1}\right)$, that is, because the reflection point is not affected by the counterflow at prime tense. With the disturbances caused by counterflow spread downstream, the value of $\alpha_{\text {ref }}\left(M_{0}\right)$ decreases sharply, even below the von Neumann shock angle (see region $\mathrm{k}_{2}$ ). After turning off the counterflow (symbol C), the shock configuration near the slot changes greatly. In company with the disturbances spread downstream, 


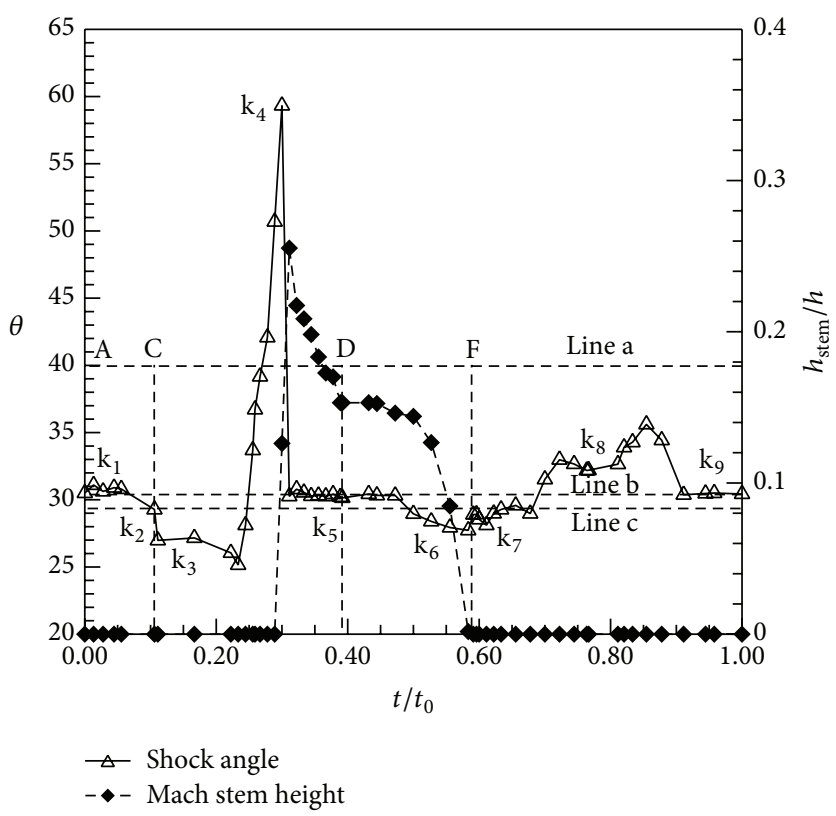

FIGURE 11: Curves of the incident shock angle at the reflection point and Mach stem height during the process.

the incident shock angle at reflection point $\mathrm{P}$ decreases smoothly at first and increases sharply then, shown in region $\mathrm{k}_{3}$. A few times later, $\alpha_{\text {ref }}\left(M_{0}\right)$ exceeds the detached angle and reaches a peak value subsequently. Then, $\alpha_{\text {ref }}\left(M_{0}\right)$ falls to the initial value $\alpha_{w}\left(M_{0}\right)$ rapidly, from the peak value (see regions $\mathrm{k}_{4}$ and $\left.\mathrm{k}_{5}\right)$. It should be noted that the value of $\alpha_{\text {ref }}\left(M_{0}\right)$ is always beyond the von Neumann angle $\alpha^{N}\left(M_{0}\right)$ during the falling process. Thus, the flow field of the hypersonic inlets after stabilization contains a stable MR configuration, finally. In a word, the detached criterion played a dominant role during this transition from RR to MR in the hypersonic inlets.

Similarly to the transition from RR to MR, counterflow is also applied for realizing the transition from MR to RR. From region $\mathrm{k}_{6}$ shown in Figure 11, the incident shock angle at reflection point $\mathrm{P}$ remains $\alpha_{w}\left(M_{0}\right)$, before the reflection region is affected by counterflow. Once the disturbances spread into the reflection point, $\alpha_{\text {ref }}\left(M_{0}\right)$ decreases below the von Neumann angle soon. At the same time, the height of Mach stem decreases gradually, until it disappears. After turning off the counterflow, the incident shock angle at reflection point increases beyond the von Neumann angle. However, the value of $\alpha_{\text {ref }}\left(M_{0}\right)$ is always below the detached angle, during the stabilization of the flow field in the hypersonic inlets. Thus, the final shock reflection pattern in the hypersonic inlets is RR configuration. During this transition, the von Neumann criterion plays a dominant role.

3.4. Evolution of Shock Reflection Configuration. Figure 12 illustrated contour lines of the Mach number in a hypersonic inlet, to reveal the evolution of flow field during the transition from RR to MR. Combining the characters of wave configurations, it is evident to divide the process into three stages, transmission of waves, emergence of Mach stem, and stabilization of flow field.

At the first stage, the wave configurations in the hypersonic inlet are changed when the counterflow is turned off. And the disturbance spreads downstream gradually. Further, the flow field shown in Figure 12(a) indicates the incident shock is composed by an oblique shock with an angle at $\alpha_{w}\left(M_{0}\right)$ and a curved shock. Besides, the curved shock angle at reflection point $\mathrm{P}$ is much smaller than the oblique shock angle at junction $\mathrm{c}$. With the disturbance spread downstream, the incident shock angle at reflection point $\mathrm{P}$ increases rapidly. During the stage, the Mach stem does not appear, even though $\alpha_{w}\left(M_{0}\right)$ is beyond the detached shock, slightly.

At the second stage, with the above angle exceeding the detached angle sharply, the Mach stem appeared. Shortly, the curved shock became a part of Mach stem, because of the durative growing of shock angle at the reflection point. It also could explain why the Mach stem height increased sharply at $t / t_{0} \approx 0.2-0.3$ in Figure 11 .

Juxtaposing Figures 12(e) and 12(f), the continual reduction of Mach stem height is the chief character for this stage. Finally, the height of Mach stem remains invariable.

\section{Conclusion}

Experimental results show that there exist two different flow fields when the forebody waves interact with the lip boundary in the hypersonic inlets. Further analysis indicates the phenomenon may be related to the shock reflection hysteresis phenomenon. In order to improve the performance of the flow field, counterflow is applied to control the shock reflection configuration in this paper. For better understanding of the internal mechanism, inviscid numerical simulation is conducted and following conclusions could be obtained.

(1) Increasing the total pressure of the counterflow results in the fact that the detached shock a grows larger in scale. Besides, increasing the velocity of the counterflow makes the reflection point $\mathrm{P}$ move downstream.

(2) By adding the counterflow, it is feasible to realize the transition between the regular reflection configuration and the Mach reflection configuration in the hypersonic inlet.

(3) The nature of flow control process is related to the shock reflection hysteresis. During the transition from RR to MR, the detached criterion plays a dominant role. However, during the transition from MR to RR, the von Neumann criterion plays a dominant role.

(4) The evolution of flow field during the transition from RR to MR could be divided into three stages, which are referred to as transmission of waves, emergence of Mach stem, and stabilization of flow field. 


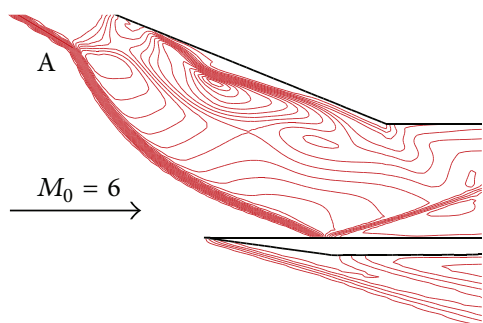

(a) $t=1.05 \times 10^{-3}$

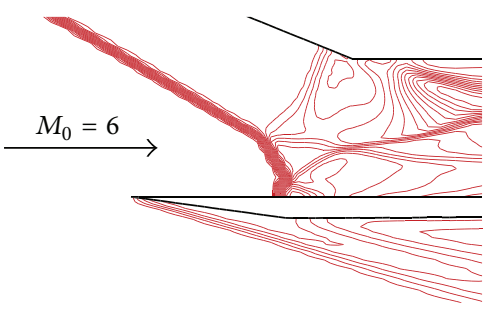

(d) $t=1.35 \times 10^{-3}$

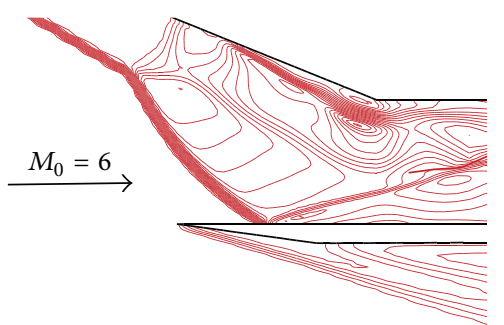

(b) $t=1.15 \times 10^{-3}$

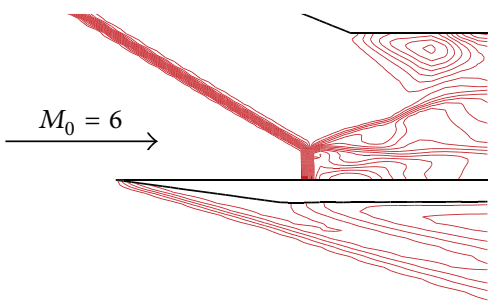

(e) $t=1.45 \times 10^{-3}$

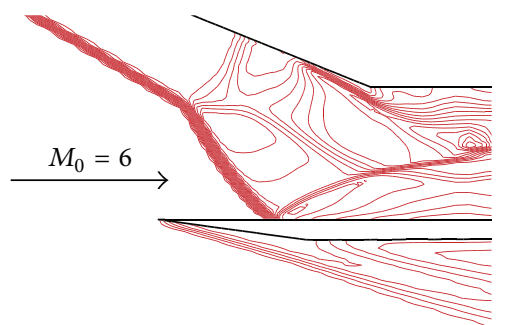

(c) $t=1.25 \times 10^{-3}$

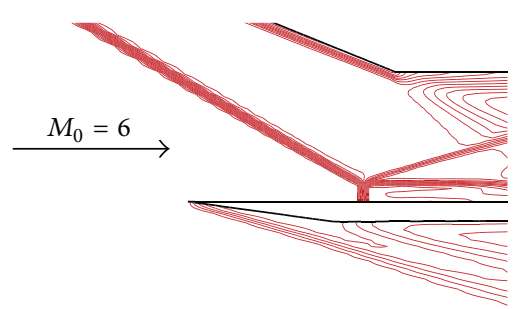

(f) $t=1.75 \times 10^{-3}$

FIGURE 12: The evolution of flow field during the transition from RR to MR.

\section{Conflict of Interests}

The authors declare that there is no conflict of interests regarding the publication of this paper.

\section{Acknowledgments}

The authors would like to gratefully acknowledge the help of Science and Technology on Scramjet Laboratory. This work was supported by National Natural Science Foundation of China NSFC Grant no. 11372347. This support was gratefully acknowledged.

\section{References}

[1] C. Segal, The Scramjet Engine Processes and Characteristics, Cambridge University Press, New York, NY, USA, 2009.

[2] G. Ben-Dor, Shock Wave Reflection Phenomena, Shock Wave and High Pressure Phenomena, Springer, New York, NY, USA, 2nd edition, 2007.

[3] Y. Tao, X. Fan, and Y. Zhao, "Flow visualization for the evolution of the slipstream in steady shock reflection," Journal of Visualization, vol. 18, no. 1, pp. 21-24, 2015.

[4] H. G. Hornung, H. Oertel, and R. J. Sandeman, "Transition to Mach reflexion of shock waves in steady and pseudosteady flow with and without relaxation," Journal of Fluid Mechanics, vol. 90, no. 3, pp. 541-560, 1979.

[5] A. Chpoun, D. Passerel, H. Li, and G. Ben-Dor, "Reconsideration of oblique shock wave reflections in steady flows. Part 1. Experimental investigation," Journal of Fluid Mechanics, vol. 301, pp. 19-35, 1995.

[6] M. S. Ivanov, A. N. Kudryavtsev, S. B. Nikiforov, D. V. Khotyanovsky, and A. A. Pavlov, "Experiments on shock wave reflection transition and hysteresis in low-noise wind tunnel," Physics of Fluids, vol. 15, no. 6, pp. 1807-1810, 2003.

[7] M. S. Ivanov, G. N. Markelov, A. N. Kudryavtsev, and S. F. Gimelshein, "Numerical analysis of shock wave reflection transition in steady flows," AIAA Journal, vol. 36, no. 11, pp. 2079-2086, 1998.

[8] C.-J. Tam, K.-C. Lin, D. L. Davis, and R. Behdadnia, "Numerical investigations on simple variable geometry for improving scramjet isolator performance," in Proceedings of the 42nd AIAA/ASME/SAE/ASEE Joint Propulsion Conference \& Exhibit, AIAA Paper 4509-2006, Sacramento, Calif, USA, July 2006.

[9] J. Moss and E. Perrell, "Design and preliminary analysis of variable geometry SCRAM jet/rocket," in Proceedings of the 40th AIAA/ASME/SAE/ASEE Joint Propulsion Conference and Exhibit, AIAA Paper 2004-3652, Fort Lauderdale, Fla, USA, July 2004.

[10] F. Falempin, E. Wendling, M. Goldfeld, and A. Starov, "Experimental investigation of starting process for a variable geometry air inlet operating from Mach 2 to Mach 8," in Proceedings of the 42nd AIAA/ASME/SAE/ASEE Joint Propulsion Conference \& Exhibit, AIAA 2006-4513, Sacramento, Calif, USA, 2006.

[11] H. Yan, R. Adelgren, G. Elliott, T. Beutner, and M. Ivanov, "Laser energy deposition in quiescent air and intersecting shocks," in Proceedings of the 4th Workshop on Magneto-Plasma Aerodynamics for Aerospace Applications, Moscow, Russia, 2002.

[12] H. Yan, R. Adelgren, G. Elliott, D. Knight, and T. Beutner, "Effect of energy addition on MR $\rightarrow$ RR transition," Shock Waves, vol. 13, no. 2, pp. 113-121, 2003.

[13] H. Yan, R. Adelgreny, G. Elliottz, D. Knight, T. Beutner, and M. Ivanov, "Laser energy deposition in intersecting shocks," in Proceedings of the 1st Flow Control Conference, St. Louis, Miss, USA, June 2002.

[14] R. G. Adelpen, H. Yan, G. S. Elliott et al., "Localized flow control by laser energy deposition applied to Edney IV shock impingement and intersecting shocks," in Proceedings of the 41st Aerospace Sciences Meeting and Exhibit, AIAA 2003-31, Reno, Nev, USA, January 2003.

[15] M. N. Shneider, S. O. Macheret, S. H. Zaidi, I. G. Girgis, and R. B. Miles, "Virtual shapes in supersonic flow control with energy addition," Journal of Propulsion and Power, vol. 24, no. 5, pp. 900-915, 2008. 
[16] T. A. Lapushkina, S. V. Bobashev, R. V. Vasil'eva et al., "Influence of electric and magnetic fields on the shock wave configuration at the diffuser inlet," Technical Physics, vol. 47, no. 4, pp. 397-405, 2002.

[17] J. Syberg and L. Koncsek, "Bleed system design technology for supersonic inlets," AIAA 72-1138, 1972. 

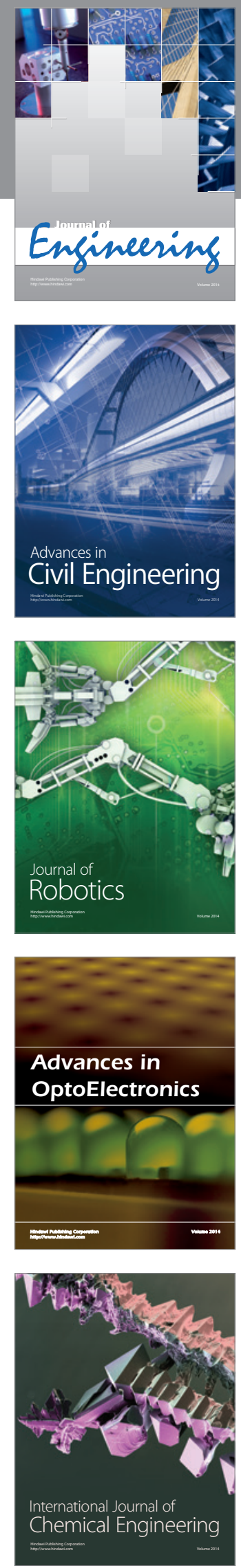

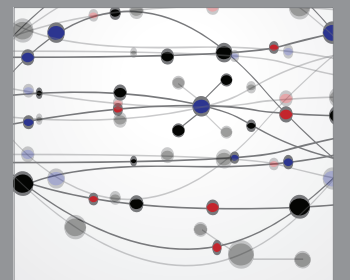

The Scientific World Journal
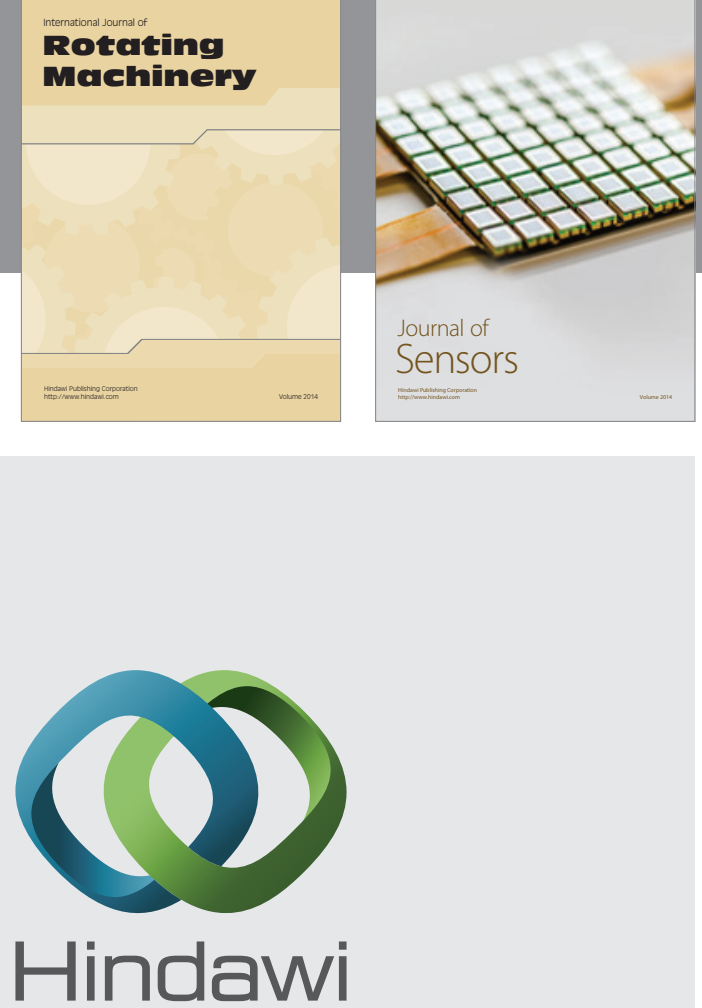

Submit your manuscripts at http://www.hindawi.com
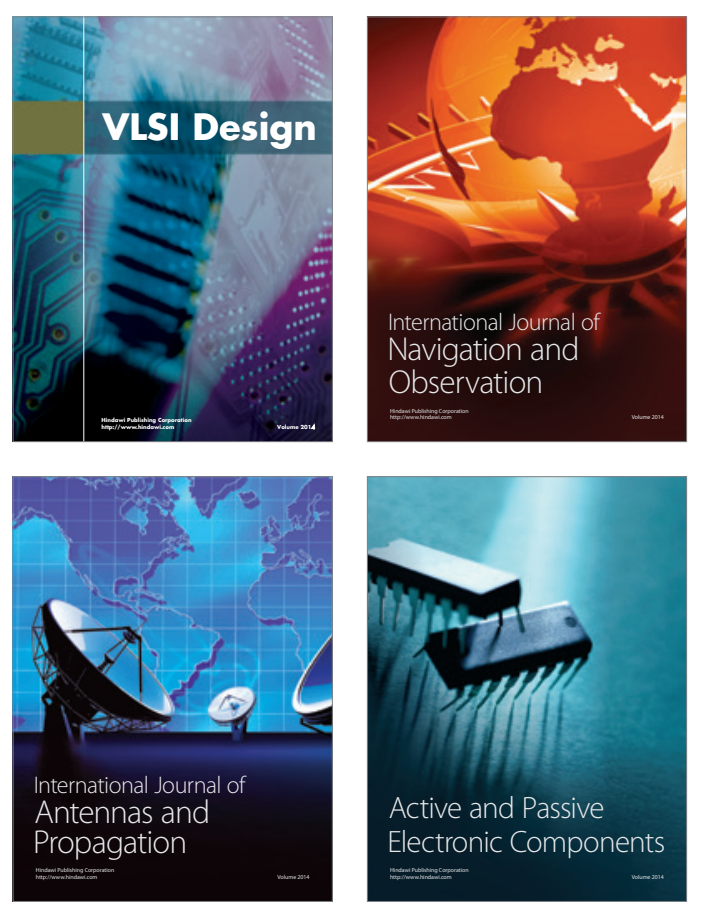
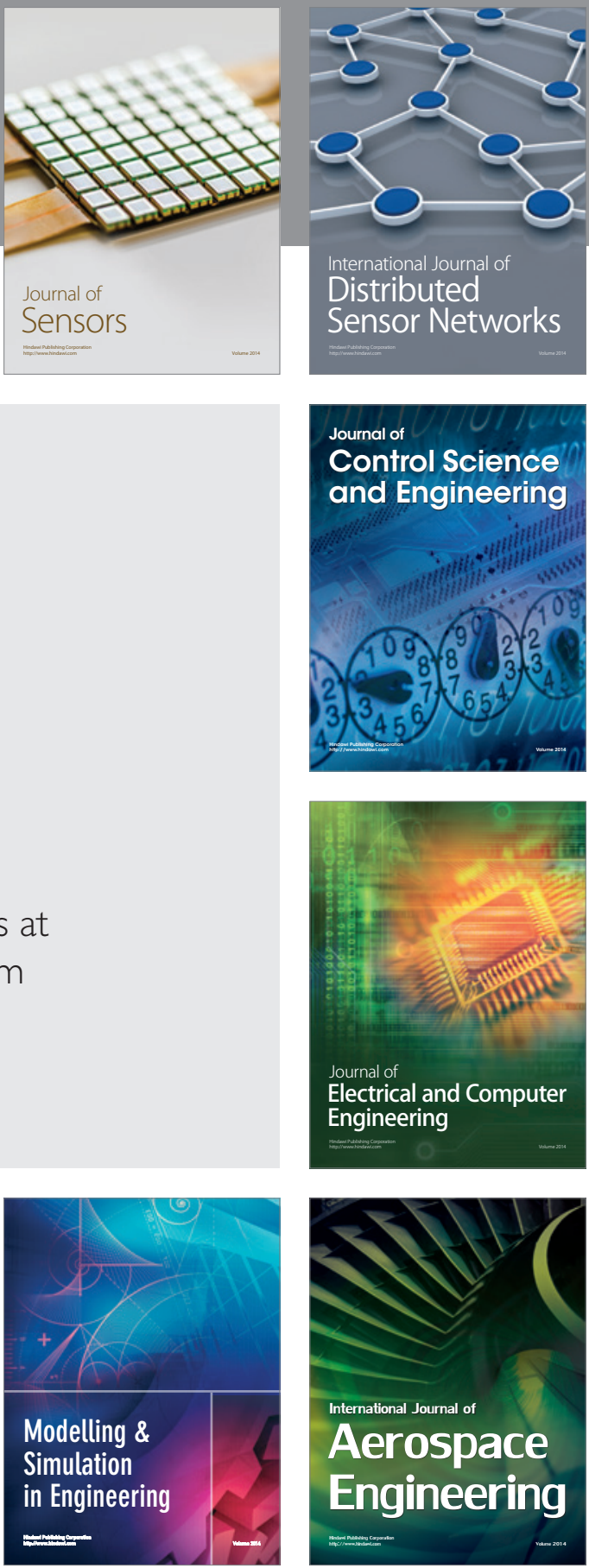

Journal of

Control Science

and Engineering
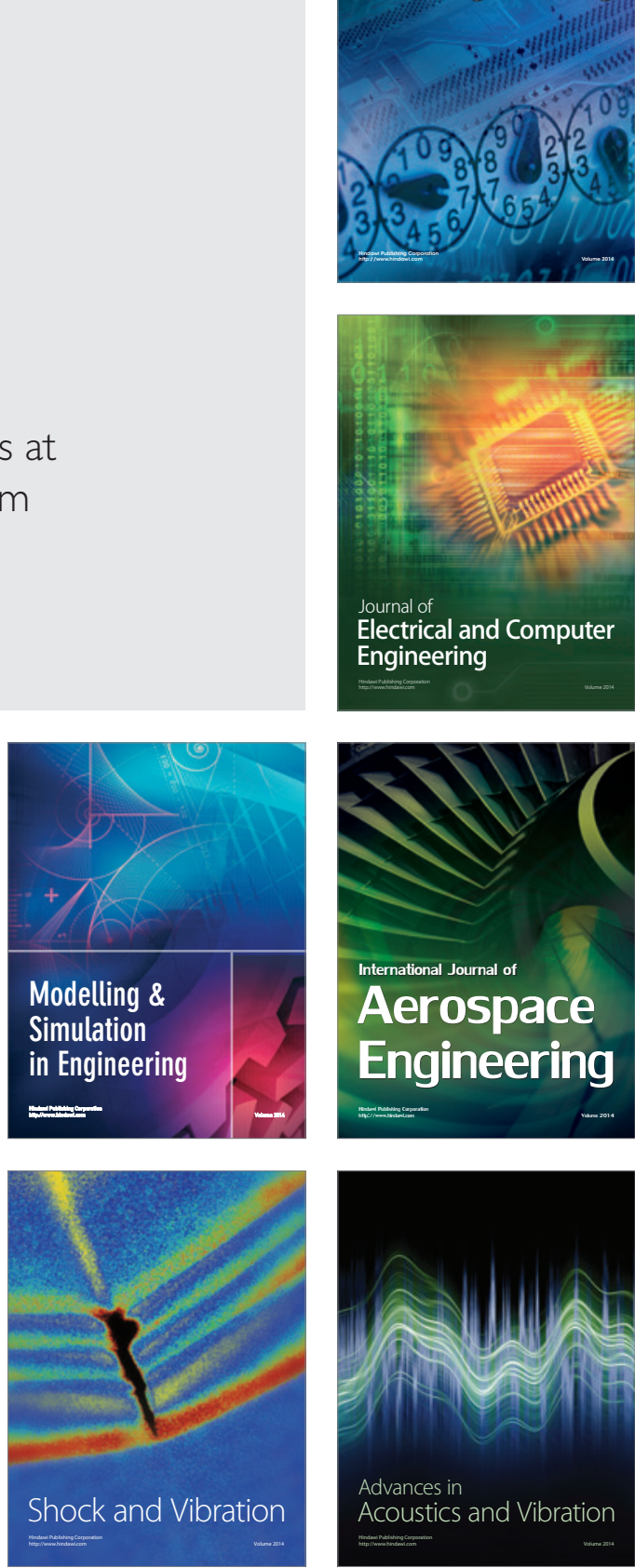